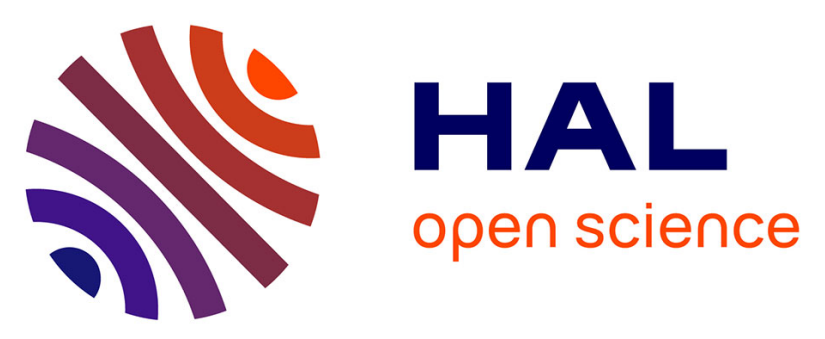

\title{
Phenotype-based drug screening in primary ovarian carcinoma cultures identifies intracellular iron depletion as a promising strategy for cancer treatment
} Joachim Gullbo, Mårten Fryknäs, Linda Rickardson, Padraig Darcy, Maria Hägg, Malin Wickström, Sadia Hassan, Gunnar Westman, Slavica Brnjic, Peter Nygren, et al.

\section{To cite this version:}

Joachim Gullbo, Mårten Fryknäs, Linda Rickardson, Padraig Darcy, Maria Hägg, et al.. Phenotype-based drug screening in primary ovarian carcinoma cultures identifies intracellular iron depletion as a promising strategy for cancer treatment. Biochemical Pharmacology, 2011, 10.1016/j.bcp.2011.04.003 . hal-00703549

\section{HAL Id: hal-00703549 \\ https://hal.science/hal-00703549}

Submitted on 3 Jun 2012

HAL is a multi-disciplinary open access archive for the deposit and dissemination of scientific research documents, whether they are published or not. The documents may come from teaching and research institutions in France or abroad, or from public or private research centers.
L'archive ouverte pluridisciplinaire HAL, est destinée au dépôt et à la diffusion de documents scientifiques de niveau recherche, publiés ou non, émanant des établissements d'enseignement et de recherche français ou étrangers, des laboratoires publics ou privés. 


\section{Accepted Manuscript}

Title: Phenotype-based drug screening in primary ovarian carcinoma cultures identifies intracellular iron depletion as a promising strategy for cancer treatment

Authors: Joachim Gullbo, Mårten Fryknäs, Linda Rickardson, Padraig Darcy, Maria Hägg, Malin Wickström, Sadia Hassan,

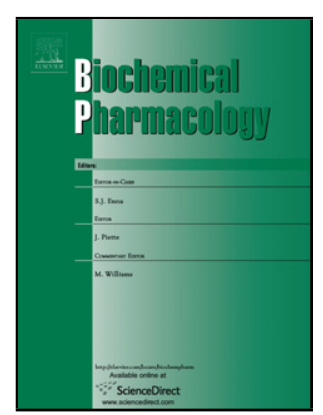

Gunnar Westman, Slavica Brnjic, Peter Nygren, Stig Linder,

Rolf Larsson

PII:

S0006-2952(11)00242-5

DOI: doi:10.1016/j.bcp.2011.04.003

Reference:

BCP 10875

To appear in: $\quad B C P$

Received date: 21-2-2011

Revised date: $\quad$ 7-4-2011

Accepted date: $\quad 14-4-2011$

Please cite this article as: Gullbo J, Fryknäs M, Rickardson L, Darcy P, Hägg M, Wickström M, Hassan S, Westman G, Brnjic S, Nygren P, Linder S, Larsson R, Phenotype-based drug screening in primary ovarian carcinoma cultures identifies intracellular iron depletion as a promising strategy for cancer treatment, Biochemical Pharmacology (2010), doi:10.1016/j.bcp.2011.04.003

This is a PDF file of an unedited manuscript that has been accepted for publication. As a service to our customers we are providing this early version of the manuscript. The manuscript will undergo copyediting, typesetting, and review of the resulting proof before it is published in its final form. Please note that during the production process errors may be discovered which could affect the content, and all legal disclaimers that apply to the journal pertain. 
Phenotype-based drug screening in primary ovarian carcinoma cultures identifies intracellular iron depletion as a promising strategy for cancer treatment

Joachim Gullbo ${ }^{1,6}$, Mårten Fryknäs ${ }^{1,3,6}$, Linda Rickardson ${ }^{1}$, Padraig Darcy ${ }^{3}$, Maria Hägg ${ }^{3}$, Malin Wickström ${ }^{1}$, Sadia Hassan ${ }^{1}$, Gunnar Westman ${ }^{4}$, Slavica Brnjic ${ }^{3}$, Peter Nygren ${ }^{2}$, Stig Linder ${ }^{3}$, and Rolf Larsson ${ }^{1,5}$

${ }^{1}$ Department of Medical Sciences, Division of Clinical Pharmacology, Uppsala University Hospital, S-751 85 Uppsala, Sweden; ${ }^{2}$ Department of Oncology, Radiology and Clinical Immunology, Uppsala University, S-751 85 Uppsala, Sweden; ${ }^{3}$ Cancer Center Karolinska, Karolinska Institute, Stockholm, ${ }^{4}$ Department of Chemical and Biological Engineering, Chalmers University of Technology, S-412 96 Gothenburg, Sweden

${ }^{5}$ Corresponding author. Email: rolf.larsson@medsci.uu.se

Fax: +4618519237 Phone: +46186115098

${ }^{6}$ Contributed equally to this work

Running title: Iron depletion as a therapeutic strategy for solid tumors Key Words: Screening, Ovarian Cancer, Primary Cultures, Iron Depletion 


\begin{abstract}
Primary cultures of patient tumor cells (PCPTC) have been used for prediction of diagnosis-specific activity and individual patient response to anticancer drugs, but have not been utilized as a model for identification of novel drugs in high throughput screening. In the present study ovarian carcinoma cells from three patients were tested in response to a library of 3000 chemically diverse compounds. Eight hits were retrieved after counter screening using normal epithelial cells, and one of two structurally related hit compounds was selected for further preclinical evaluation. This compound, designated VLX 50, demonstrated a broad spectrum of activity when tested in a panel of PCPTCs representing different forms of leukemia and solid tumors and displayed a high tumor to normal cell activity. VLX 50 induced delayed cell death with some features of classical apoptosis. Significant in vivo activity was confirmed on primary cultures of human ovarian carcinoma cells in mice using the hollow fiber model. Mechanistic exploration was performed using gene expression analysis of drug exposed tumor cells to generate a drug-specific signature. This query signature was analyzed using the Gene Set Enrichment Analysis and the Connectivity Map database. Strong connections to hypoxia inducible factor 1 and iron chelators were retrieved. The mechanistic hypothesis of intracellular iron depletion leading to hypoxia signaling was confirmed by a series of experiments. The results indicate the feasibility of using PCPTC for cancer drug screening and that intracellular iron depletion could be a potentially important strategy for cancer therapy.
\end{abstract}




\section{INTRODUCTION}

During the past decades most screening approaches for identification of new cancer drug candidates have utilized cell free assays for detection of specific interactions with known or emerging molecular targets [1]. However, the relatively poor outcome with respect to identification of clinically novel and significantly improved cancer drugs has led to a renewed and growing interest for cancer drug screening based on compound induced changes in cellular phenotypes [2]. Monolayer cultures of human tumor cell lines have been the general model in these efforts and are important tools for predicting mechanisms of drug action as demonstrated in numerous reports [3,4]. Furthermore, recent results utilizing very large panels of cell lines indicate that they also to a large extent retain genomic features of the primary tumor and can recapitulate clinical findings with regard to their response to targeted inhibitors [4].

Primary cultures of patient tumor cells (PCPTC) is an alternative cell based tumor model system that has received very little attention in the context of cancer drug screening and development. Most of the research efforts using PCPTC models have focused on prediction of clinical activity of cancer drugs for individual patients $[5,6]$. However, nonclonogenic in vitro assays performed on PCPTC from different diagnoses can detect tumor-type specific activity of standard [7] and investigational cancer drugs [8, 9]. Thus, PCPTC should constitute a potentially valuable tool for preclinical drug development of cancer drugs.

In the beginning of the 1980s the applicability of a human tumor colony-forming assay using fresh tumor biopsy-specimens from patients was investigated by an NCIcoordinated study as a potential alternative or complement to cell line screening [10]. This system demonstrated sensitivity to most standard agents, discriminated non-toxic compounds and provided evidence for the capability to detect active compounds which were negative in the former P388 mouse leukemia in vivo screening [10]. Nevertheless, due to technical limitations inherent in the assay methodology the program using primary specimens was not pursued any further. More recently we investigated the feasibility of using a short-term assay for cancer drug screening using PCPTCs from ovarian carcinoma [3]. These cultures readily discriminated between clinically active and non- 
toxic agents and selected a higher fraction of solid tumor active agents when compared to established tumor cell lines [3]. Although apparently feasible, utility of PCPTC in primary screening will ultimately rely on their ability to detect clinically active drugs not detected in cell lines as well as solving practical (e.g. access to PCPTC, cell requirements and inter-individual variability), and ethical limitations. A brief review on the bioassay test methods developed for high throughput screening assays using both cellbased and molecular approaches, was published recently [11]. While cell line models are associated with specific limitations, it has been predicted that future anti-cancer drug development is likely to use a combination of molecular, cell line, primary or early passage cell culture, and xenograft methods for lead optimisation before clinical trials are contemplated [12].

In the present study PCPTCs of ovarian carcinoma were tested in response to 3,000 chemically diverse compounds. Eight confirmed hits were retrieved and after retest and counter screening using normal epithelial hTERT-RPE1 cells, one (CD 02750 subsequently denoted VLX 50) out of two structurally related hit compounds was selected for mechanistic investigation and further preclinical evaluation. 


\section{EXPERIMENTAL PROCEDURES}

\subsection{Cell culture}

For primary screening three PCPTCs from previously chemotherapy treated ovarian carcinoma were used. An additional 96 PCPTCs from different tumor types, and four preparations of normal peripheral blood mononuclear cells (PBMC), detailed in Table 1, were used to determine the activity spectrum of VLX 50 and, for comparison, six standard cytotoxic drugs chosen to represent different mechanistic classes. Tumor cells of ovarian carcinoma were obtained from ascites fluid using standard techniques for tumor cell isolation described in detail previously [13]. Malignant cells from hematological and solid tumors were obtained as described previously [14, 15], and briefly in supplementary information section 1.1. The patient sampling was approved by the local ethics committee at Uppsala university hospital.

The human cell lines used for mechanistic studies were breast cancer MCF7 (breast cancer), hTERT-RPE1 (normal epithelial cell line), HepG2 (hepatocellular carcinoma), HCT116 (colon carcinoma), and A549 (non-small cell lung cancer). MCF7 and hTERTRPE1 were obtained from American Type Culture Collection (ATCC) and Clontech (Palo Alto, CA), respectively. HepG2 was obtained from ECACC (Salisbury, UK). All experiments on purchased cell lines were performed with less than 6 months passages in our laboratory after receipt or resuscitation. All cell lines were sub-cultivated and grown in supplemented medium as recommended by the providers.

\subsection{Preparation of compounds for screening}

The Maybridge Hitskit 3000 library (Maybridge Inc.) consists of 3000 chemically diverse compounds. The content of this library is described in supplementary table S2. The library was delivered in 36 racks each containing 80 compounds dissolved in DMSO to $10 \mathrm{mg} / \mathrm{ml}$. For the screening, four different 384-well plates were prepared with final test concentrations of $1 \mu \mathrm{g} / \mathrm{ml}$. For concentration-response studies, plates were prepared by 10 -fold serial dilutions in the concentrations 0.004 to $40 \mu \mathrm{M}$.

\subsection{Measurement of cancer drug activity}


The Fluorometric Microculture Cytotoxicity Assay, FMCA, described in detail recently [16] was used for measurement of the cytotoxic effect of library compounds and the established standard drugs. Cells were exposed during 72 hours in 384-well microtiter plates in a standard incubator, washed and cell viability was assayed by addition of fluorescein diacetate (details in supplementary information, section 1.2).

\subsection{Multiparametric high content evaluation of apoptosis, cell cycle arrest, and oxidative stress}

The fluorescence microscope ArrayScan II HCS system (Cellomics Inc., Pittsburgh, PA, USA) was used to study apoptosis, cell cycle arrest and oxidative stress. For these assays cells were seeded into 96-well plates (PerkinElmer Inc., Wellesley, MA, USA), left to attach over night, before test compounds were added. Apoptosis was evaluated after 6, 24 and $48 \mathrm{~h}$ exposure to VLX 50 in MCF-7 cells by fluorescent staining of nuclei (Hoechst) and caspase 3 (FAM-DEVD). Cell cycle and oxidative stress were assayed after $24 \mathrm{~h}$ exposure with commercially available kits (Cell Cycle Kit I, and Oxidative Stress I Kit, respectively, Thermo Fisher Scientific, Gothenburg, Sweden) as described by the manufacturer. More details of methodology and reagents used are given in supplementary information (section 1.4).

\subsection{Phase contrast microscopy}

Time-lapse phase contrast microscopy was performed using an automated IncuCyte phase contrast microscope (Essen Instruments, Ann Arbor, MI). MCF-7 cells (10 000/well) were plated on 24-well ImageLock plates (Essen Instruments, Ann Arbor, MI) and cultured in complete medium.

\subsection{Microarray analysis and qPCR}

Connectivity Map (cmap) build 02 contains genome-wide expression data for more than 1300 compounds (6 100 instances, including replicates, different doses and cell lines). The original protocol using MCF-7 breast cancer cells as described by Lamb et al. was used [17]. Expression of selected genes was confirmed with quantitative Polymerase Chain Reaction (qPCR), all details are given in supplementary information (section 1.6).

\subsection{Measurements of iron concentration}


The green-fluorescent heavy metal indicator Phen Green SK dipotassium salt (Invitrogen $\mathrm{AB}$, Gothenburg, Sweden) was used to measure iron concentration differences in a solution. Details are given in supplementary information (section 1.7).

\subsection{Measurements of ribonucleotide reductase activity}

Ten million $\left(1 \times 10^{7}\right)$ HCT-116 cells grown in $150 \mathrm{~mm}$ plates were exposed to indicated drug for $24 \mathrm{~h}$. The ribonucleotide assay was performed as previously described [18], with modification as detailed in supplementary information (section 1.8).

\subsection{In vivo studies}

PCPTC from two ovarian carcinoma patients and the T-cell leukemia cell line CCRFCEM were cultured inside semi-permeable polyvinylidene fluoride fibers (500 kDa molecular weight cut-off, $1 \mathrm{~mm}$ diameter; Spectrum, Laguna Hills, CA) and assessed in the hollow fiber assay $[19,20]$. The fibers were implanted subcutaneously into the back of immunocompetent animals (male NMRI mice, Scanbur, Sollentuna Sweden), which were treated once with $0.76 \mathrm{mg} /$ mouse of VLX 50 , or vehicle only ( $\mathrm{n}=8$ animals/group). Dose was selected after a preliminary dose-finding study showing intolerable toxicity (transient effects on locomotor activity, hunched position, and convulsions) at 1.9 $\mathrm{mg}$ /mouse (unpublished data). Fibers were retrieved after 6 days and cell density evaluated using the MTT assay [21], details are given in supplementary information (section 1.9). The study was approved by the Animal Ethics Committee in Uppsala, Sweden.

\subsection{Data analysis and statistics}

Small Laboratory Information and Management System, SLIMS [22] was used for screening data management. Raw fluorescence data files were loaded into the SLIMS software which calculates percent inhibition according to the formula: Percent inhibition $=100 \mathrm{x}$ (x-negative control/positive control-negative control) -1 , where $\mathrm{x}$ denotes fluorescence from experimental wells. SLIMS also identifies and corrects systematic spatial errors. Screening data was subsequently exported to Vortex (Dotmatics Inc, UK) software for further analysis. A Survival Index of less than $50 \%$ in ovarian carcinoma PCPTCs $(n=3)$ was set as the criteria for qualifying as hit compound. Structural similarity 
to other compounds in the library was calculated based on a structural fingerprint consisting of chemical fragments located within the compound and which are computed for each compound by Vortex. Clustering of compounds based on these fingerprints was then performed within the Vortex software.

The Z'-value was calculated to evaluate the quality and usefulness of the assay in the screening setting using the equation:

$Z^{\prime}=1-\left[\left(3 \mathrm{SD}_{\text {poscontrol }}+3 \mathrm{SD}_{\text {negcontrol }}\right) /\left(\mathrm{Mean}_{\text {poscontrol }}-\mathrm{Mean}_{\text {negcontrol }}\right)\right]$.

where SD and mean are the standard deviation and mean values of screening raw data from wells with untreated cells (positive control) and blank wells (negative control), respectively [23].

Concentration-response data of screening hits and standard agents were analyzed with GraphPadPrism4 (GraphPad Software Inc., San Diego, CA, USA), using non-linear regression to a standard sigmoidal dose-response model to obtain $\mathrm{IC}_{50}$-values (concentration resulting in $\mathrm{SI}=50 \%$ ).

Response rate in PCPTCs of a specific diagnosis was defined as the fraction of samples having an SI below the median, calculated from all PCPTCs included in the study, at the drug concentration showing the largest SD. For VLX 50 this concentration was $4 \mu \mathrm{M}$. The data for the reference compound imatinib was taken from Lindhagen et al [24], and recalculated as response rate at $1 \mu \mathrm{M}$ (sample origin in supplementary table S1). The relative effect of a drug on solid compared with hematological tumors was indicated by the $\mathrm{S} / \mathrm{H}$ ratio, defined as the ratio between the total response rates for the solid and the hematological samples. Tumor cell specific activity was estimated by calculation of the ratio of the median $\mathrm{IC}_{50}$-value for PBMC over that of chronic lymphocytic leukemia (CLL) samples.

Comparisons between groups in the hollow fiber experiment were done with Student's ttest. 


\section{RESULTS}

\subsection{Drug screening in PCPTC of ovarian cancer identifies novel hit compounds} The ovarian carcinoma PCPTC ( $n=3)$ grew as 3-D clusters (Fig. 1A) and were tested for cytotoxic/antiproliferative activity in response to 3000 chemically diverse compounds at a concentration of $1 \mu \mathrm{g} / \mathrm{ml}$ using FMCA. All 3 PCPTCs were screened separately and were not mixed. Eight confirmed hits giving more than 50\% cell kill were retrieved $(0.2 \%$ hit rate, Fig. 1B). All hits are presented in supplementary table S5 (inhibition values, molecular weights and structures). The assay was shown to be simple and robust, and the Z'-value of the assay using PCPTC was 0.44 , which indicates an acceptable variability and dynamic range. Clustering of the compounds according to chemical structure revealed that two of the hit compounds, CD 02750 (most active, hereafter denoted VLX 50) and CD 02862, were of similar chemical structure (Fig. 1C-D). Five of the eight hits based on mean percent inhibition fulfilled the hit criteria in all 3 PCPTC samples, including VLX 50. For VLX 50 the individual values were 71.6, 72.4 and $66.9 \%$, respectively. Two other compounds belonging to the same chemical cluster (CD02749, CD01914) lacked activity at $1 \mu \mathrm{g} / \mathrm{ml}$ (Fig. 1B). After retesting in additional ovarian cancer PCPTCs $(n=14)$ and counter screening using normal epithelial hTERTRPE1 cells, it was shown that VLX 50 exhibited tumor-selective activity (no effect against hTERT-RPE1 cells at $10 \mu \mathrm{M}, \mathrm{IC}_{50}$-difference approximately 10 -fold) and was therefore selected for further preclinical evaluation (Fig. 2A).

\subsection{Diagnosis-specific activity of VLX50 ex vivo suggest activity in lymphocytic malignancies, ovarian and breast cancer}

To further examine the activity spectrum of VLX 50, its cytotoxic effect of was studied in 96 tumor samples from patients with a variety of solid tumors and hematological malignancies as well as in four samples of primary healthy lymphocytes (PBMC). Median $\mathrm{IC}_{50}$-values ranged from less than $5 \mu \mathrm{M}$ for diagnoses such as chronic lymphocytic leukemia (CLL), acute lymphocytic leukemia (ALL), ovarian carcinoma and lymphoma to above $40 \mu \mathrm{M}$ for colon and renal cancer samples (Table 1). Cancer of the breast, lung, chronic myelocytic leukemia (CML), acute myelocytic leukemia (AML) and PBMC displayed intermediate sensitivity to VLX 50. The in vitro response rates to VLX 50 at $4 \mu \mathrm{M}$ for the PCPTC of various diagnoses is listed in Fig. 2B. Consistent with the 
$\mathrm{IC}_{50}$ patterns the lymphocytic malignancies showed the highest response rates followed by ovarian carcinoma and breast cancer whereas PBMC, colon and renal cancer demonstrated the lowest response rates.

The tyrosine kinase inhibitor imatinib was included as a reference compound demonstrating a very narrow activity spectrum with CML samples showing the highest in vitro response rate at the selected test concentration of $1 \mu \mathrm{M}$ (Fig. 2B).

The relative effect, expressed as the S/H ratio, of VLX 50 and six standard cytotoxic drugs, in solid and hematological tumor samples, expressed as the $\mathrm{S} / \mathrm{H}$ ratio is shown in Fig. 2C. VLX 50 had a ratio of 0.73 indicating a relatively high activity against solid tumors second only to cisplatin ( $\mathrm{S} / \mathrm{H}$ ratio 1.2). The remaining drugs showed $\mathrm{S} / \mathrm{H}$ ratios below 0.5 . The results for the standard drugs are consistent with their main clinical use. To roughly estimate tumor cell specificity, drug effects were compared in cells from CLL and normal PBMCs. VLX 50 demonstrated a significantly higher activity against the malignant phenotype with a PBMC/CLL median $\mathrm{IC}_{50}$ ratio of 7.6 (Fig. 2D). Of the tested standard cytotoxic drugs only vincristine was more active in CLL than in PBMC.

\subsection{VLX50 significantly inhibits in vivo growth of ovarian PCPTC}

In vivo activity of VLX 50 was investigated in hollow fiber cultures of PCPTC from ovarian carcinoma patients subcutaneously implanted in mice ( $\mathrm{n}=8$ each in test and control groups, total $\mathrm{n}=16$ ). After a single dose VLX 50 of $760 \mu \mathrm{g} /$ mouse significant growth inhibition compared to vehicle treatment were observed in the two PCPTC cultures ( $p<0.05$ and $p<0.01$, respectively, Fig. $3 A$ and B). In comparison, the difference did not reach statistical significance in the acute lymphoblastic leukemia cell line CCRF CEM (p>0.05, Fig. 3C). VLX 50 induced a small but significant reduction in weight gain compared to the control group (start weight 32.7 vs 34.8 , end weight 32.5 vs $35.5 \mathrm{~g}$, $\mathrm{p}<0.05$, not shown). A significant decrease in platelet counts was also observed (1022 vs $\left.1310 \times 10^{9} / \mathrm{L}, \mathrm{p}<0.05\right)$. No effects on white blood cell count (WBC), red blood cell count (RBC) or haemoglobin values were noted ( $p>0.05$, not shown).

\subsection{VLX 50 induces apoptosis in cancer cells}

Next we examined the mode of cell death caused by VLX 50 in MCF-7 cells using timelapse phase contrast microscopy and multi-parameter analysis for apoptosis using 
Arrayscan II. The activity of VLX 50 on growth and viability was delayed in time with little or no effect observable at $6 \mathrm{~h}$, but with a concentration dependent effect on cell growth over time (Fig. 4A). At 24-48 h there was a gradual decrease in cell density and a parallel increase in DNA fragmentation and caspase-3 activity (Fig. 4B and C). Phase contrast images of the cells at this time point revealed typical apoptotic morphology with condensed nuclei surrounded by a bright halo (Fig. 4A). Moreover, MCF-7 cells treated with $10 \mu \mathrm{M}$ VLX 50 showed annexin V positivity (Supplementary Fig. S3), and a time dependent increase in caspase cleaved cytotokeratin 18 (Supplementary Fig. S4). These data taken together suggests, at least partly, the involvement of caspase-dependent apoptosis in mediating cell death induced by VLX 50.

\subsection{VLX 50 is identified as an iron depleting agent}

Mechanistic exploration was performed by measurement of gene expression of drug treated tumor cell cultures. The breast cancer cell line MCF-7 was exposed to VLX 50 or vehicle (DMSO) for 6 hours followed by microarray based gene expression analysis. A drug specific query signature was generated based on the 30 most up and down regulated genes. This query signature was subsequently uploaded to the Connectivity Map (cmap), to find other compounds with similar mechanism of action. The VLX50 signature showed strongest similarity to 2-hydroxy-3-methoxybenzaldehyde pyridin-2-ylhydrazone, ciclopiroxamine, and deferoxamine, all known to be potent iron chelators [25-27] (Fig. 5A). This suggested that VLX 50's mechanism of action is iron chelation. Gene set Enrichment analysis (GSEA) of genes induced by VLX 50 [25] showed strong connection to genes and genes up regulated under hypoxia and hypoxia inducible factor 1 alpha (HIF1 $\alpha$ ) target (Fig. 5B). This response is consistent with iron chelation.

The mechanistic hypothesis of VLX 50 causing intracellular iron depletion was tested by adding $100 \mu \mathrm{M}$ iron (as $\mathrm{FeCl}_{3}$ ) to VLX 50 exposed MCF-7 cell cultures which resulted in a significant decrease of VLX 50 cytotoxic activity (Fig. 5C), an effect that was dosedependent regarding iron supplement (not shown). Addition of iron also had effects on the cytotoxicity of the known iron chelator ciclopirox (Fig 6C), but not of docetaxel and doxorubicin included as reference substances (shown in supplementary information only, Fig S1). The mechanism was further confirmed by direct measurements of drug induced decrease in iron concentration as measured by the de-quenching of the metal ion probe, 
Phen Green (Fig. 5D). The VLX50:Fe complex is yellow and can be detected visually or spectrophotometrically (supplementary Fig. S2). Genes associated with HIF1 signaling were up regulated by VLX 50 (Fig. 5B and supplementary table S4). The experiment was repeated and quantified with qPCR, and again PDK1, HK2, NDRG1, VEGFA and the genes encoding the apoptosis associated BH3-only proteins BNIP3 and BNIP3L were induced in a dose dependent manner (Fig. 6A). VLX 50 induced accumulation of cells in G1 as judged by the DNA content (Fig. 6B). No detectable increase in Phospho histone $\mathrm{H} 3$, typical of G2/M arrest, was observed (supplementary information), in concordance with other iron chelators. There was no increase in oxidative stress response as compared to the positive control rotenone (Fig. 6C). Finally, VLX 50 concentration dependently inhibited the iron-dependent enzyme ribonucleotide reductase at concentrations associated with tumor cell death (Fig. 6D).

\section{DISCUSSION}

Although tumor cell line models, as the NCI60 and other panels, can provide important information on mechanisms of action of anticancer drugs, the correlation between the activity in cell lines and in patients with the corresponding tumor types is not well documented and screening for cancer activity using PCPTC could potentially be an alternative [3,28]. In vitro response analysis of PCPTCs reports accurate diagnosis specific activity of cancer drugs [7] and predict clinical response for individual patients [6]. This model system would thus be expected to provide clinically relevant information on the activity of potentially novel cancer drugs. In the present study this ability is indicated by the activity spectra of the tyrosine kinase inhibitor imatinib which was correctly identified as drug with relatively unique activity in CML.

In this study, a chemically diverse compound library was screened using PCPTC from ovarian carcinoma. Only $0.2 \%$ of the compounds were identified as hits using $50 \%$ percent inhibition at $1 \mu \mathrm{g} / \mathrm{ml}$ as the cut-off criterion. This hit rate is clearly lower compared to that observed in cell lines [29]. This may be due to several factors. First, PCPTC represents low proliferative system thus being less sensitive to drug induced cell cycle perturbations. Second, PCPTC cells are cultured as 3-dimensional clusters which 
are known to be more resistant to drug effects compared to monolayer cultures due, not only to pharmacokinetic obstacles limiting drug penetrance to inner layers, but also to multi-cellular interactions leading to altered expression of genes and proteins regulating drug response [30]. Third, the concentration selected for screening was $1 \mu \mathrm{g} / \mathrm{ml}$ which for most of the compounds in the library is below the standard $10 \mu \mathrm{M}$ normally used for cancer drug screening. Finally, PCPTC culture may have a different compound recognition ability compared to established human tumor cell lines continuously propagated in culture.

It is generally believed that cell lines cultured for multiple passages may loose many phenotypic features characteristics of histological origin [31] and different drug recognition patterns between human tumor cell lines and PCPTC have been suggested previously [3]. Indeed supporting the latter, VLX 50 and its structurally related analogue showed only modest activity in high proliferative cell line systems (Supplementary table S3). However, it should be noted that in a complementary ten cell line screen, VLX 50 fulfilled the hit criteria in two of the cell lines although not ranked among the top 15 compounds. Thus, PCPTC seem to have the ability to detect drug activity not obviously apparent in the small cell line panel used here (see supplementary table S3). It can not, however, be excluded that employing a much larger cell line panels would have detected VLX 50 as a significant hit [4]. Finally, in the context of screening ovarian carcinoma is especially well suited as a PCPT model of solid tumors since very large numbers of cells $\left(>10^{9}\right)$ can easily be obtained from ascites fluid of a single patient.

VLX 50 demonstrated a broad spectrum of activity in PCPTCs of various tumor types second only to cisplatin with respect to relative solid tumor activity. Moreover, the high PBMC/CLL $\mathrm{IC}_{50}$ ratio indicates a potentially high therapeutic index ex vivo. It should be emphasized that both the PBMC/CLL ratio and $\mathrm{S} / \mathrm{H}$ ratios are in vitro indicators for therapeutic index and clinical activity spectra and should be evaluated in relative rather than absolute terms. A ratio of 1 indicates equal sensitivity for PBMC vs CLL and solid vs hematological activity, respectively. Thus, comparing and ranking different drugs with the respect to these measures is a preferable way to use these indices. Indeed, $\mathrm{S} / \mathrm{H}$ index have previously been shown to correlate well to the clinical activity profile of standard cytotoxic cancer agents [7]. Furthermore, supporting these ex vivo findings VLX 50 had 
significant in vivo activity against patient ovarian carcinoma cells at modest toxicity indicating favorable pharmacokinetics and pharmacodynamic properties in vivo. It should be noted that the hollow fiber is a very resistant in vivo tumor model requiring the drug to penetrate into fibers implanted deep subcutaneously thus yielding a low false positive rate for cancer activity in vivo compared with other in vivo models [32]. No significant activity could be detected in the high proliferating leukemia cell line, which once again suggests that the compound has some selectivity for the PCPTC.

For exploration of mechanisms of action we used a bioinformatic approach using a drug specific gene expression signature to probe the cmap and GSEA databases. The cmap data base indicated strong connections to iron chelators whereas GSEA connected the signature to hypoxia and HIF1 $\alpha$ signaling. These results strongly suggest that VLX 50 induces intracellular iron depletion, which subsequently leads to HIF $1 \alpha$ mediated hypoxia signaling. Indeed, addition of extracellular iron abolishes the effect of VLX 50 and the drug was shown to effectively decrease free iron in solution further confirming this notion. The fact that VLX 50 was the most potent of the four semithiocarbazones in the library (Fig. 1) is probably due to the basic nitrogen of the 2-substituted pyridyl ring, which contributes positively to coordination of the iron ion in a tridentate complex [33].

Cancer cells have a higher requirement for iron than normal cells as reflected by increased numbers of transferrin receptors and increased ferritin content in tumor tissues [26]. The higher demand for iron may at least partly be explained by the increased activity of iron dependent enzymes such as ribonucleotide reductase (RR), a rate-limiting step in DNA synthesis [34]. Indeed it has also been demonstrated that the activity and expression of RR is increased in tumor cells indicating a high level DNA synthesis in these cells [35]. However, the activation of HIF $1 \alpha$ mediated transcription may also contribute to cell death mediated by iron depletion [36]. HIF $1 \alpha$ is the regulatory and DNA binding subunit of the HIF1 transcription factor, which under conditions of adequate oxygen supply is hydroxylated by the Fe-containing enzyme prolyl hydroxylase leading to proteasomal degradation and diminished transcriptional activity [36]. However, under hypoxic conditions or iron depletion prolyl hydroxylase is rendered inactive resulting in increased transcription of HIF $1 \alpha$ regulated genes. Notable in this context is the HIF1 $\alpha$ dependent increase in expression of the apoptosis inducing gene 
BNIP3 [37] and the growth and metastasis suppressor NDRG-1 [38]. VLX 50 significantly increased the expression, supporting the potential involvement of these genes. In addition iron depletion may lead to differential expression of a range of cell cycle molecules including cyclin D1-3, p21 and CDK2 which may contribute to the G1/S arrest observed after iron depletion induced by VLX 50 and other iron chelators $[39,40]$.

Since iron chelators have previously been reported to induce cell death through the inhibition of RR this principle could provide an effective mechanistic principle for cancer drugs. Desferoxamine is an extracellular iron chelator currently used in the clinic for treatment of iron overload disorders [26]. In addition desferoxamine has also demonstrated anti-proliferative activity against a wide variety of tumor cells and anticancer activity has been reported in clinical trials [41, 42]. More recently, the intracellular iron chelator 3-aminopyridine-2-carboxaldehyde thiosemicarbazone (Triapine) has been developed as a potential anticancer agent with an excellent preclinical activity in many tumor models and is currently undergoing Phase I and II clinical trials $[26,43,44]$. Triapine has been shown to be a potent inhibitor of RR [44]. However, in addition to RR inhibition Triapine has been reported to be redox active leading to ROS formation [45] potentially adding to the reported drug induced toxicity. ROS generation could lead to several toxicological consequences as a result of oxidative injury to important biomolecules such as DNA, proteins and lipids [33]. In contrast to Triapine, VLX 50, although belonging to the thiosemicarbzone group of compounds, did not appear to induce ROS formation. This could be a distinct advantage in the clinical setting. However the optimal properties and role of iron chelators in the treatment of cancer remains to be determined.

In conclusion, the present study indicates the feasibility of using PCPTC for drug screening and that intracellular iron depletion could be a promising mechanistic pathway to explore in the development of more active cancer drugs.

\section{ACKNOWLEDGEMENT}

We are grateful for all the work with microarray array analysis at the Uppsala Expression Array Platform. The skillful technical assistance of Christina Leek and Lena Lenhammar 
is gratefully acknowledged. This study was supported by the Swedish Cancer Society (S.L., P.N., and R.L.), the Swedish Research Council (S.L.) and the Lions Cancer Research Fund (P.N. and R.L.). 


\section{REFERENCES}

[1] Fox S, Farr-Jones S, Sopchak L, Boggs A, Nicely HW, Khoury R, et al. High-throughput screening: update on practices and success. J Biomol Screen 2006;11:864-9.

[2] Hart CP. Finding the target after screening the phenotype. Drug Discov Today 2005;10:513-9.

[3] Dhar S, Gullbo J, Nilsson K, Nygren P, Larsson R. A nonclonogenic cytotoxicity assay using primary cultures of patient tumor cells for anticancer drug screening. J Biomol Screen 1998;3:207-15.

[4] Sharma SV, Haber DA, Settleman J. Cell line-based platforms to evaluate the therapeutic efficacy of candidate anticancer agents. Nat Rev Cancer 2010;10:241-53.

[5] Cree IA. Chemosensitivity and chemoresistance testing in ovarian cancer. Curr Opin Obstet Gynecol 2009;21:39-43.

[6] Nygren P, Larsson R. Predictive tests for individualization of pharmacological cancer treatment. Expert Opin Med Diagn 2008;2:1-12.

[7] Fridborg H, Jonsson E, Nygren P, Larsson R. Relationship between diagnosis-specific activity of cytotoxic drugs in fresh human tumour cells ex vivo and in the clinic. Eur J Cancer 1999;35:424-32.

[8] Wickstrom M, Danielsson K, Rickardson L, Gullbo J, Nygren P, Isaksson $\mathrm{A}$, et al. Pharmacological profiling of disulfiram using human tumor cell lines and human tumor cells from patients. Biochem Pharmacol 2007;73:25-33.

[9] Wickstrom M, Haglund C, Lindman H, Nygren P, Larsson R, Gullbo J. The novel alkylating prodrug $\mathrm{J1}$ : diagnosis directed activity profile ex vivo and combination analyses in vitro. Invest New Drugs 2008;26:195-204.

[10] Shoemaker RH, Wolpert-DeFilippes MK, Kern DH, Lieber MM, Makuch RW, Melnick NR, et al. Application of a human tumor colony-forming assay to new drug screening. Cancer Res 1985;45:2145-53.

[11] Harvey AL, Cree IA. High-throughput screening of natural products for cancer therapy. Planta Med 76:1080-6.

[12] Cree IA, Glaysher S, Harvey AL. Efficacy of anti-cancer agents in cell lines versus human primary tumour tissue. Curr Opin Pharmacol 10:375-9.

[13] Csoka K, Tholander B, Gerdin E, de la Torre M, Larsson R, Nygren P. In vitro determination of cytotoxic drug response in ovarian carcinoma using the fluorometric microculture cytotoxicity assay (FMCA). Int $\mathrm{J}$ Cancer 1997;72:1008-12.

[14] Larsson R, Kristensen J, Sandberg C, Nygren P. Laboratory determination of chemotherapeutic drug resistance in tumor cells from patients with leukemia, using a fluorometric microculture cytotoxicity assay (FMCA). Int J Cancer 1992;50:177-85.

[15] Csoka K, Larsson R, Tholander B, Gerdin E, de la Torre M, Nygren P. Cytotoxic drug sensitivity testing of tumor cells from patients with ovarian carcinoma using the fluorometric microculture cytotoxicity assay (FMCA). Gynecol Oncol 1994;54:163-70. 
[16] Lindhagen E, Nygren P, Larsson R. The fluorometric microculture cytotoxicity assay. Nat Protoc 2008;3:1364-9.

[17] Lamb J, Crawford ED, Peck D, Modell JW, Blat IC, Wrobel MJ, et al. The Connectivity Map: using gene-expression signatures to connect small molecules, genes, and disease. Science 2006;313:1929-35.

[18] Jong AY, Yu K, Zhou B, Frgala T, Reynolds CP, Yen Y. A simple and sensitive ribonucleotide reductase assay. J Biomed Sci 1998;5:62-8.

[19] Friberg LE, Hassan SB, Lindhagen E, Larsson R, Karlsson MO. Pharmacokinetic-pharmacodynamic modelling of the schedule-dependent effect of the anti-cancer agent CHS 828 in a rat hollow fibre model. Eur $\mathrm{J}$ Pharm Sci 2005;25:163-73.

[20] Jonsson E, Friberg LE, Karlsson MO, Hassan SB, Freijs A, Hansen K, et al. Determination of drug effect on tumour cells, host animal toxicity and drug pharmacokinetics in a hollow-fibre model in rats. Cancer Chemother Pharmacol 2000;46:493-500.

[21] Scudiero DA, Shoemaker RH, Paull KD, Monks A, Tierney S, Nofziger TH, et al. Evaluation of a soluble tetrazolium/formazan assay for cell growth and drug sensitivity in culture using human and other tumor cell lines. Cancer Res 1988;48:4827-33.

[22] Kelley BP, Lunn MR, Root DE, Flaherty SP, Martino AM, Stockwell BR. A flexible data analysis tool for chemical genetic screens. Chem Biol 2004;11:1495-503.

[23] Zhang JH, Chung TD, Oldenburg KR. A Simple Statistical Parameter for Use in Evaluation and Validation of High Throughput Screening Assays. J Biomol Screen 1999;4:67-73.

[24] Lindhagen E, Eriksson A, Wickstrom M, Danielsson K, Grundmark B, Henriksson R, et al. Significant cytotoxic activity in vitro of the EGFR tyrosine kinase inhibitor gefitinib in acute myeloblastic leukaemia. Eur $\mathrm{J}$ Haematol 2008;81:344-53.

[25] Eberhard Y, McDermott SP, Wang X, Gronda M, Venugopal A, Wood TE, et al. Chelation of intracellular iron with the antifungal agent ciclopirox olamine induces cell death in leukemia and myeloma cells. Blood 2009;114:3064-73.

[26] Richardson DR, Kalinowski DS, Lau S, Jansson PJ, Lovejoy DB. Cancer cell iron metabolism and the development of potent iron chelators as antitumour agents. Biochim Biophys Acta 2009;1790:702-17.

[27] Tang J, Costa J, Smulders S, Molna'r G, Bousseksou A, Teat S, et al. Two-Step Spin-Transition Iron(III) Compound with a Wide [High Spin-Low Spin] Plateau. Inorg Chem 2009;48:2128-35.

[28] van Staveren WC, Solis DY, Hebrant A, Detours V, Dumont JE, Maenhaut C. Human cancer cell lines: Experimental models for cancer cells in situ? For cancer stem cells? Biochim Biophys Acta 2009;1795:92-103.

[29] Rickardson L, Fryknas M, Haglund C, Lovborg H, Nygren P, Gustafsson MG, et al. Screening of an annotated compound library for drug activity in a resistant myeloma cell line. Cancer Chemother Pharmacol 2006;58:74958. 
[30] Smalley KS, Lioni M, Herlyn M. Life isn't flat: taking cancer biology to the next dimension. In Vitro Cell Dev Biol Anim 2006;42:242-7.

[31] Voskoglou-Nomikos T, Pater JL, Seymour L. Clinical predictive value of the in vitro cell line, human xenograft, and mouse allograft preclinical cancer models. Clin Cancer Res 2003;9:4227-39.

[32] Decker S, Hollingshead M, Bonomi CA, Carter JP, Sausville EA. The hollow fibre model in cancer drug screening: the $\mathrm{NCl}$ experience. Eur $\mathrm{J}$ Cancer 2004;40:821-6.

[33] Kalinowski DS, Richardson DR. Future of toxicology--iron chelators and differing modes of action and toxicity: the changing face of iron chelation therapy. Chem Res Toxicol 2007;20:715-20.

[34] Shao J, Zhou B, Chu B, Yen Y. Ribonucleotide reductase inhibitors and future drug design. Curr Cancer Drug Targets 2006;6:409-31.

[35] Elford HL, Freese M, Passamani E, Morris HP. Ribonucleotide reductase and cell proliferation. I. Variations of ribonucleotide reductase activity with tumor growth rate in a series of rat hepatomas. J Biol Chem 1970;245:5228-33.

[36] Ke Q, Costa M. Hypoxia-inducible factor-1 (HIF-1). Mol Pharmacol 2006;70:1469-80.

[37] Chong TW, Horwitz LD, Moore JW, Sowter HM, Harris AL. A mycobacterial iron chelator, desferri-exochelin, induces hypoxia-inducible factors 1 and 2, NIP3, and vascular endothelial growth factor in cancer cell lines. Cancer Res 2002;62:6924-7.

[38] Kovacevic Z, Fu D, Richardson DR. The iron-regulated metastasis suppressor, Ndrg-1: identification of novel molecular targets. Biochim Biophys Acta 2008;1783:1981-92.

[39] Nurtjahja-Tjendraputra E, Fu D, Phang JM, Richardson DR. Iron chelation regulates cyclin D1 expression via the proteasome: a link to iron deficiency-mediated growth suppression. Blood 2007;109:4045-54.

[40] Yu Y, Kovacevic Z, Richardson DR. Tuning cell cycle regulation with an iron key. Cell Cycle 2007;6:1982-94.

[41] Donfrancesco A, De Bernardi B, Carli M, Mancini A, Nigro M, De Sio L, et al. Deferoxamine followed by cyclophosphamide, etoposide, carboplatin, thiotepa, induction regimen in advanced neuroblastoma: preliminary results. Italian Neuroblastoma Cooperative Group. Eur J Cancer 1995;31A:612-5.

[42] Donfrancesco A, Deb G, Dominici C, Pileggi D, Castello MA, Helson L. Effects of a single course of deferoxamine in neuroblastoma patients. Cancer Res 1990;50:4929-30.

[43] Chaston TB, Lovejoy DB, Watts RN, Richardson DR. Examination of the antiproliferative activity of iron chelators: multiple cellular targets and the different mechanism of action of triapine compared with desferrioxamine and the potent pyridoxal isonicotinoyl hydrazone analogue 311. Clin Cancer Res 2003;9:402-14.

[44] Finch RA, Liu M, Grill SP, Rose WC, Loomis R, Vasquez KM, et al. Triapine (3-aminopyridine-2-carboxaldehyde- thiosemicarbazone): A 
potent inhibitor of ribonucleotide reductase activity with broad spectrum antitumor activity. Biochem Pharmacol 2000;59:983-91.

[45] Shao J, Zhou B, Di Bilio AJ, Zhu L, Wang T, Qi C, et al. A FerrousTriapine complex mediates formation of reactive oxygen species that inactivate human ribonucleotide reductase. Mol Cancer Ther 2006;5:58692. 


\section{FIGURE LEGENDS}

Figure 1. Drug screening in ovarian PCPTC. A) A representative photomicrograph of PCPTC from ovarian carcinoma stained with May-Grünwald-Giemsa. B) The overall screening results (percent inhibition). Blue circles indicate the four semithiocarbazones. C) Clustering of compounds based on chemical structures using Vortex built-in algorithm showing the semithiocarbazones in blue. D) Chemical structure of the semithiocarbazones from the cluster.

Figure 2. Ex vivo activity pattern of VLX 50. A) Concentration - Effect of VLX 50 on different ovarian PCPTC $(\mathrm{n}=14)$ and on the normal epithelial cell line hTERT-RPE1 $(\mathrm{n}=4)$. Results are presented mean \pm SEM. B) The ex vivo response rate in a panel of PCPTC representing a range of diagnoses $(n=98)$ is shown for VLX 50 and the reference compound imatinib. Response rate in PCPTCs of a specific diagnosis was defined as the fraction of samples having an SI below the median, calculated from all PCPTCs included in the study at the drug concentration showing the largest variability in SI values. C) The $\mathrm{IC}_{50}$ ratio between PCPTCs from PBMC $(\mathrm{n}=4)$ and CLL $(\mathrm{n}=9)$ is shown for VLX 50 and the six standard drugs. D) The solid tumor/hematological tumor activity ratio $(\mathrm{S} / \mathrm{H}$ ratio is displayed for VLX 50 and six standard agents $(\mathrm{n}=98)$.

Figure 3. Confirmation of in vivo activity using hollow fiber cultures. The antitumoral effect of a single dose VLX 50 was investigated in mice ( $\mathrm{n}=8$ per treatment group) carrying three subcutaneous hollow fiber cultures, two ovarian carcinoma PCPTC (OC, Fig A and B) and one cell line CCRF-CEM (C). The results are presented as net growth and expressed as mean value $+\mathrm{SD}(\mathrm{n}=8)$.

Figure 4. VLX 50 induces apoptosis in the MCF-7 cell line. In panel A the kinetic effects of VLX 50 is shown using phase contrast time-lapse microscopy analyzing confluence every hour during culture in 24-well plates (left). Representative phase contrast photomicrographs of control and VLX $50(10 \mu \mathrm{M})$ exposed cultures after $72 \mathrm{~h}$ (right). Using Arrayscan II the effects of VLX 50 on average caspase-3/7 activity (B) and DNA fragmentation $(\mathrm{C})$ were evaluated and are shown over time $(6-48 \mathrm{~h})$. The results are 
expressed as percentage of the untreated control and presented as mean values $+\mathrm{SD}$ $(n=3)$.

Figure 5. VLX 50 is identified (A and $B$ ) and confirmed (C and D) as an iron chelating agent. A) Connectivity Map (cmap) results based on VLX 50 exposed MCF7 cells. Black horizontal lines in the barview represent the individual instances for the iron chelators 5109870, ciclopirox and desferoxamine. The colors represent positive (green), null (grey) and negative (red) connectivity. Score according to the cmap database. B) Gene set enrichment analysis based on VLX 50 treated MCF7 cells compared to vehicle treat control. The genes have been rank-ordered according to their relative expression. The enrichment profiles (green lines) show the enrichment of genes (represented by black vertical lines) involved in hypoxia and HIF1 signaling among the genes over-expressed in the VLX 50 treated cells (FWER p-value $<0.001$ ). C) Addition of extracellular iron (100 $\mu \mathrm{M}$ ) significantly impairs the cytotoxic activity (as $\mathrm{IC}_{50}$ ) of VLX 50 and ciclopirox in MCF-7 cells. Results are shown as mean + SD from repeated experiments $(n=2)$ where each concentration was tested in duplicate wells. No effect was seen for docetaxel and doxorubicin (see supplementary Fig S1). D) Phen Green SK fluorescence is effectively quenched by addition of $\mathrm{FeCl}_{3}$. The process could be reversed by addition of VLX 50 or ciclopirox, but not docetaxel. Representative of repeated experiments $(n=2)$ Results are presented as mean $\pm \mathrm{SD}$ from triplicate wells.

Figure 6. VLX 50 induces G1 arrest and inhibits iron dependent ribonucleotide reductase but without causing oxidative stress. A) Gene expression data (fold change) of key genes in the HIF1 signaling pathway, measured using quantitative polymerase chain reaction (qPCR) after treatment with $10 \mu \mathrm{M}$ or $50 \mu \mathrm{M}$ VLX 50. B) Effect of VLX 50 on cell cycle in HCT 116 cells as determined from DNA content (bin size $=12500$ ), representative of repeated experiments $(n=2)$. C) Effect of VLX 50 on oxidative stress in HepG2 cells measured as oxidized DHE in the cell nucleus is shown. Results are expressed as percentage of the untreated control and shown as mean $+\mathrm{SD}$ from repeated experiments $(n=2)$ where each condition was tested in duplicate wells. D) Effect of VLX 50 and the positive control ciclopirox on ribonucleotide reductase activity in HCT 116 cells. Results are expressed as percentage of the untreated control and shown as mean + SD from repeated experiments $(\mathrm{n}=2)$ where each condition was tested in duplicate wells. 
Table 1. Median IC 50 and range for different diagnoses in response to VLX 50.

\begin{tabular}{|l|c|c|c|}
\hline Diagnosis & $\begin{array}{c}\text { Median } \text { IC }_{50} \\
(\mu \mathrm{M})\end{array}$ & Range & $\mathbf{n}$ \\
\hline ALL & 0.63 & $0.055-11$ & 21 \\
\hline AML & 5.4 & $0.67-40$ & 10 \\
\hline CLL & 1.0 & $0.035-9.4$ & 9 \\
\hline CML & 5.1 & $2.1-6.4$ & 3 \\
\hline NHL & 2.3 & $0.28-40$ & 13 \\
\hline Breast cancer & 8.2 & $0.87-40$ & 7 \\
\hline Ovarian carcinoma & 3.5 & $0.26-40$ & 14 \\
\hline Lung cancer & 14 & $0.51-40$ & 6 \\
\hline Colon cancer & 40 & $0.49-40$ & 6 \\
\hline Renal cancer & 40 & $24-40$ & 7 \\
\hline PBMC & 8.9 & $2.9-13$ & 4 \\
\hline
\end{tabular}

Abbreviations. ALL, acute lymphocytic leukemia; AML, acute myelocytic leukemia; CLL, chronic lymphocytic leukemia; CML, chronic myelocytic leukemia; NHL, nonHodgkin's lymphoma; PBMC, peripheral blood mononuclear cells. 


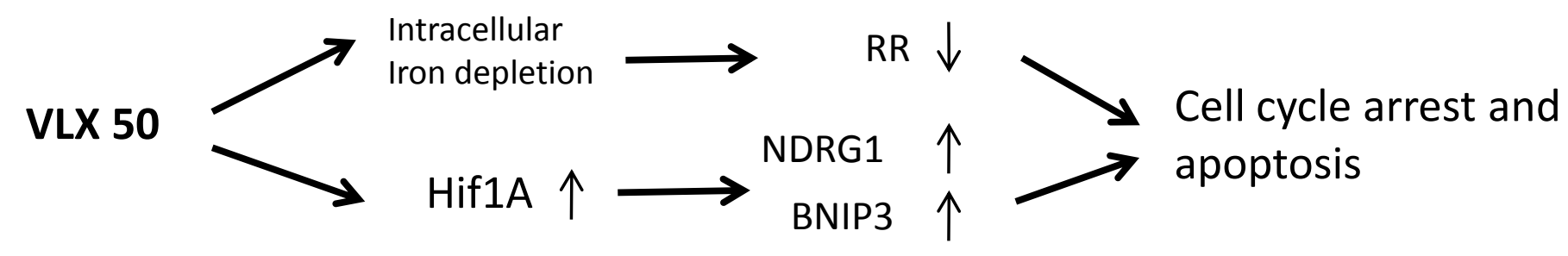

Screening primary cultures of patient tumor cells in response to a chemically diverse library of compounds identified VLX 50 as an iron chelator with tumor selective activity 
Figure 1

A

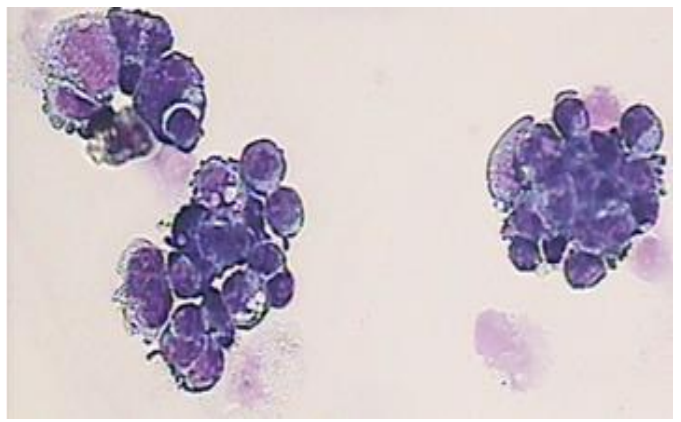

C

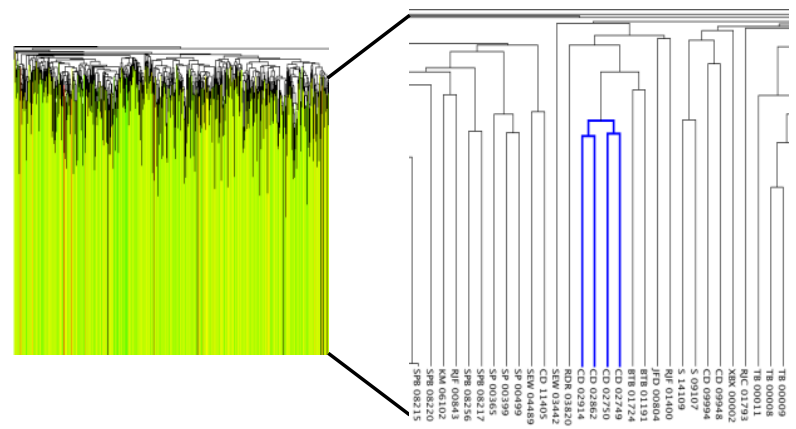

B

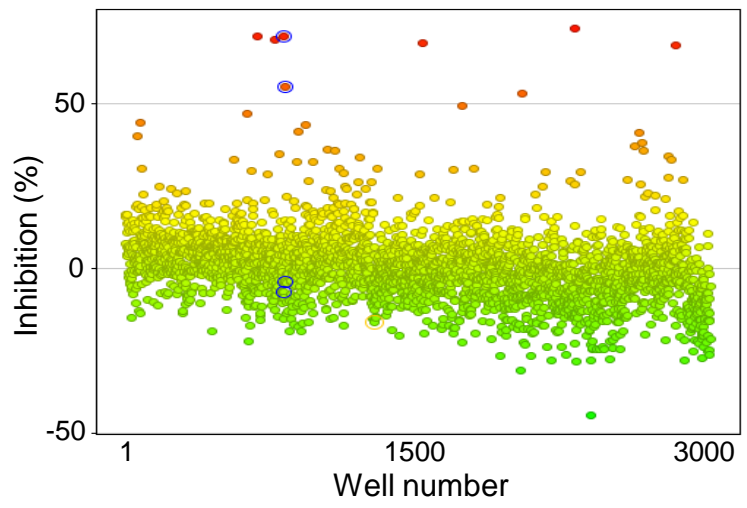

D<smiles>COCCCNC(=O)NN=Cc1ccccn1</smiles><smiles>CCOc1ccc(/C=N/NC(=O)[C@H](C)NCCCCOC)cc1</smiles><smiles>COCCCNC(=O)NNCc1ccccc1O</smiles> 
Figure 2
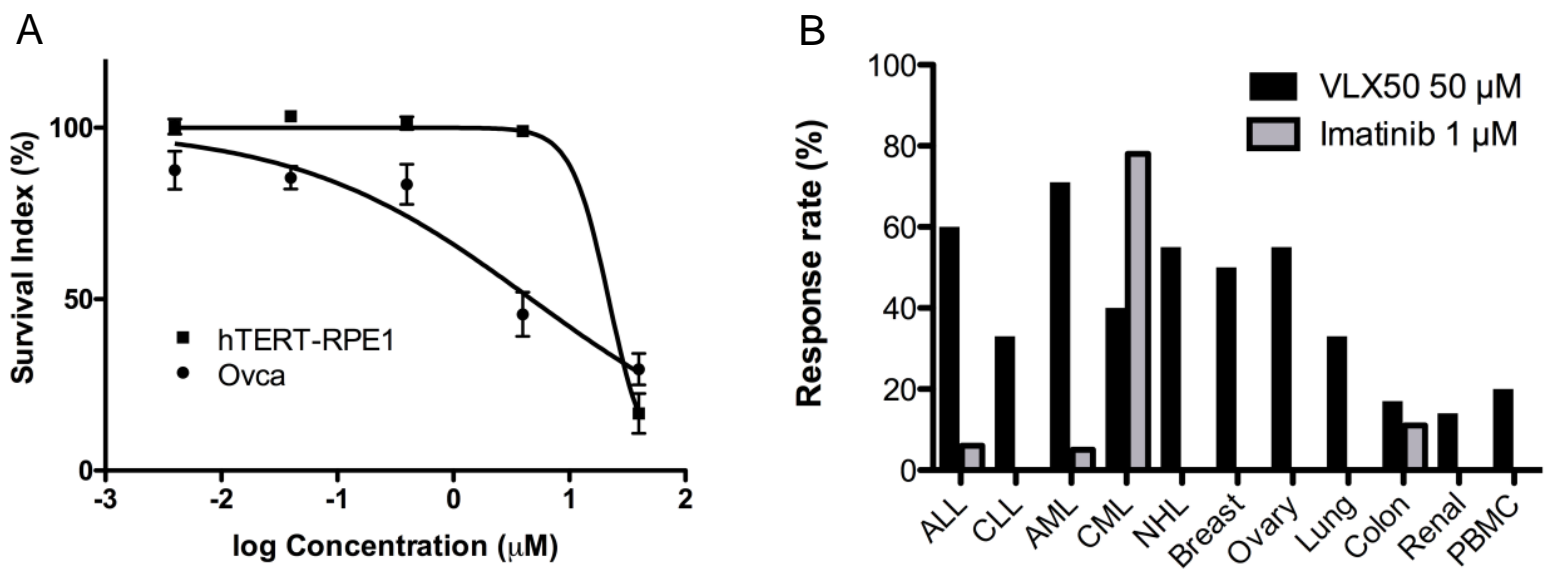

C
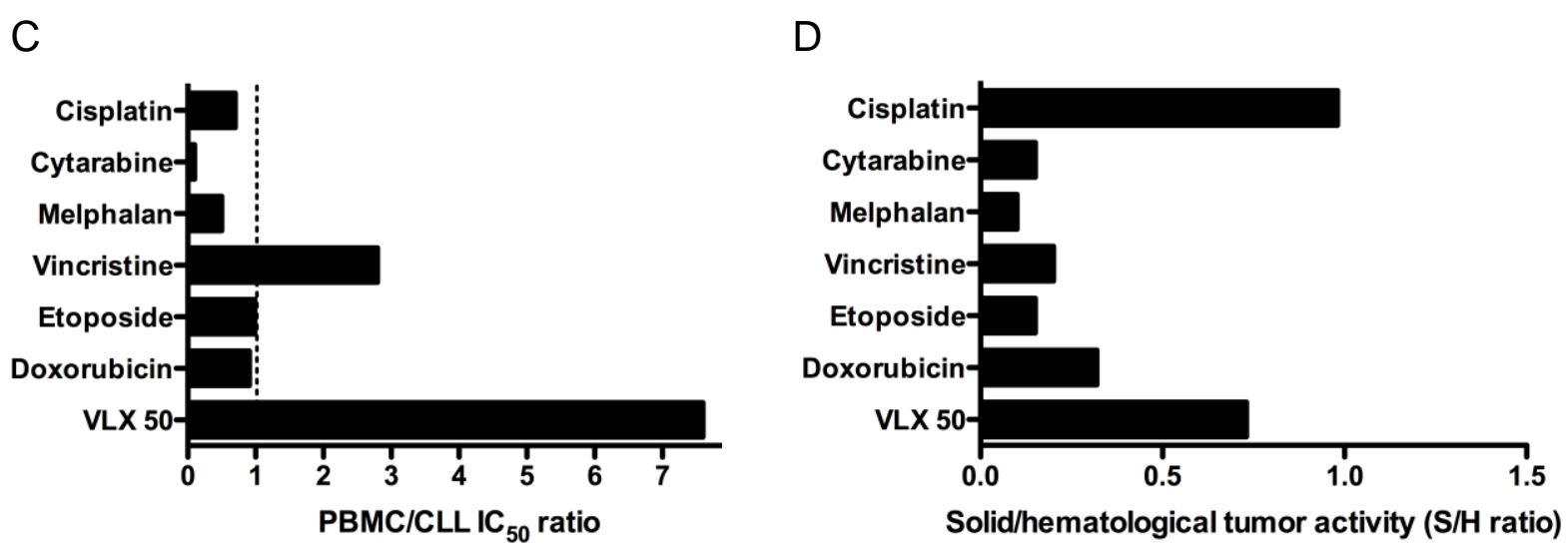

Solid/hematological tumor activity (S/H ratio) 
Figure 3

A OC1

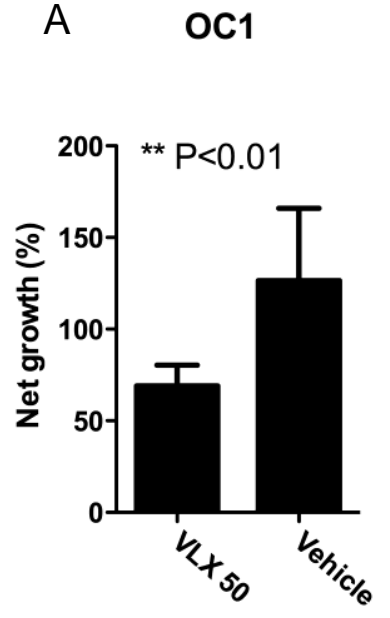

B $\quad$ Oc2

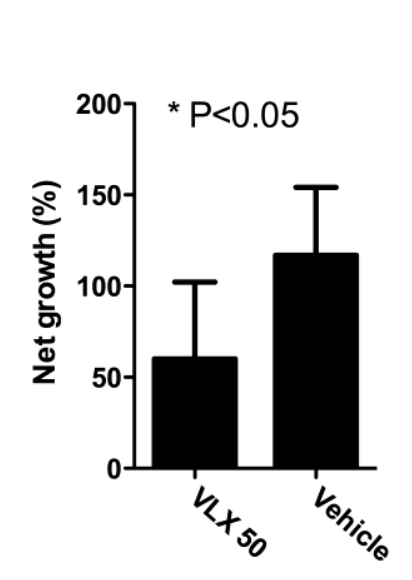

C CEM

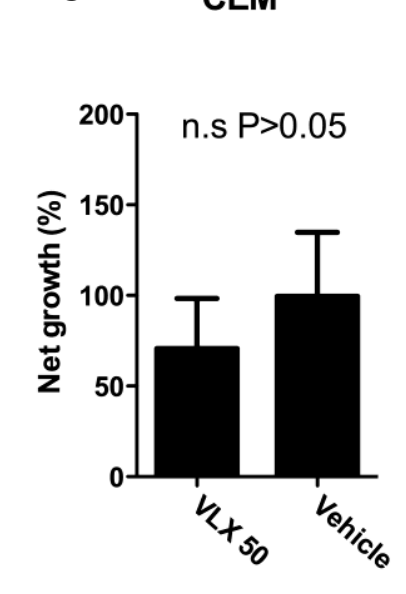


Figure 4

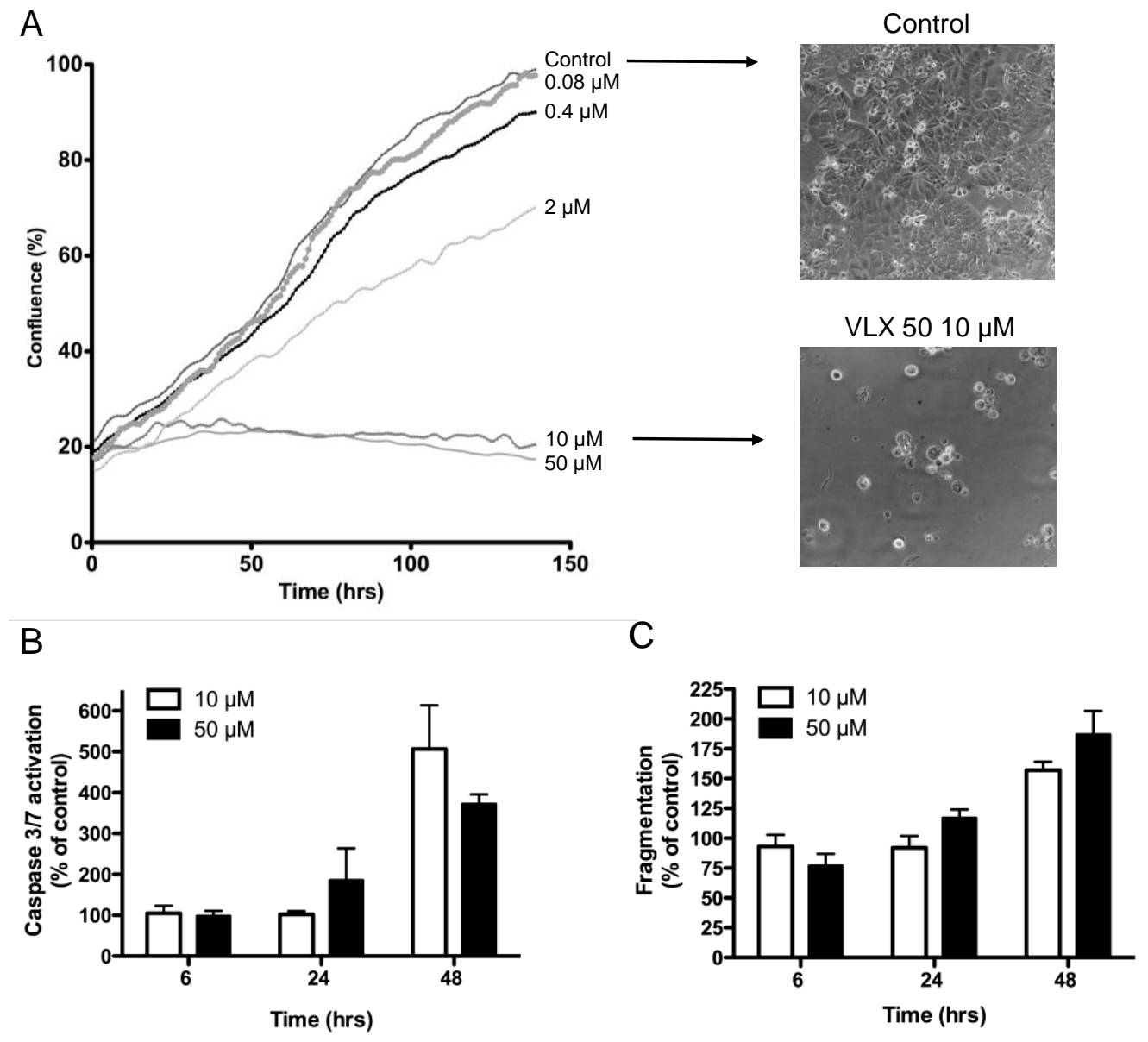


Figure 5

A

\begin{tabular}{|c|c|c|c|c|c|}
\hline \multicolumn{6}{|c|}{ Connectivety Map results, VLX 50} \\
\hline barview & rank & name & dose & cell & score \\
\hline \hline & 1 & $5109870^{*}$ & $25 \mu \mathrm{M}$ & MCF7 & 1 \\
\hline 2 & ciclopirox & $15 \mu \mathrm{M}$ & MCF7 & 0.993 \\
\hline 3 & ciclopirox & $15 \mu \mathrm{M}$ & MCF7 & 0.948 \\
\hline 4 & deferoxamine & $100 \mu \mathrm{M}$ & PC3 & 0.882 \\
\hline 20 & deferoxamine & $100 \mu \mathrm{M}$ & MCF7 & 0.771 \\
\hline 21 & ciclopirox & $15 \mu \mathrm{M}$ & PC3 & 0.771 \\
\hline 31 & deferoxamine & $100 \mu \mathrm{M}$ & MCF7 & 0.743 \\
\hline 119 & deferoxamine & $6 \mu \mathrm{M}$ & PC3 & 0.653 \\
\hline 160 & deferoxamine & $6 \mu \mathrm{M}$ & MCF7 & 0.625 \\
\hline 180 & deferoxamine & $6 \mu \mathrm{M}$ & PC3 & 0.618 \\
\hline 786 & ciclopirox & $15 \mu \mathrm{M}$ & HL60 & 0.460 \\
\hline 2260 & deferoxamine & $6 \mu \mathrm{M}$ & MCF7 & 0 \\
\hline 5261 & deferoxamine & $6 \mu \mathrm{M}$ & MCF7 & -0.337 \\
\hline
\end{tabular}

*Benzaldehyde, 2-hydroxy-3-methoxy-, 2-(2-pyridinyl)hydrazone

C

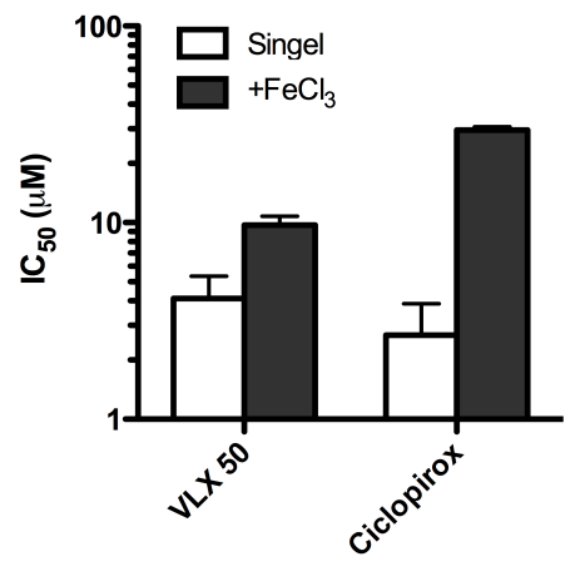

B

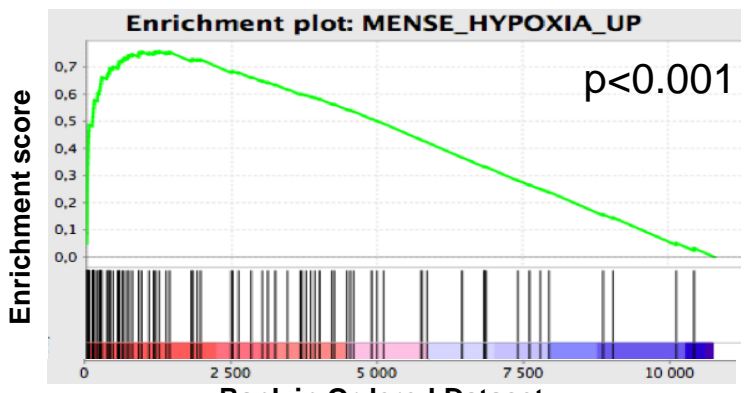

Rank in Ordered Dataset

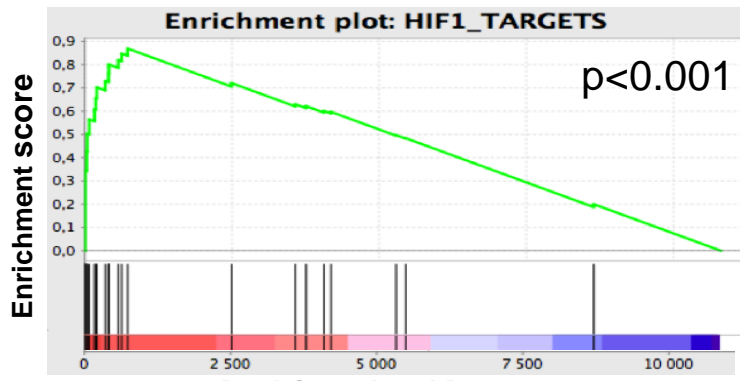

Rank in Ordered Dataset

D

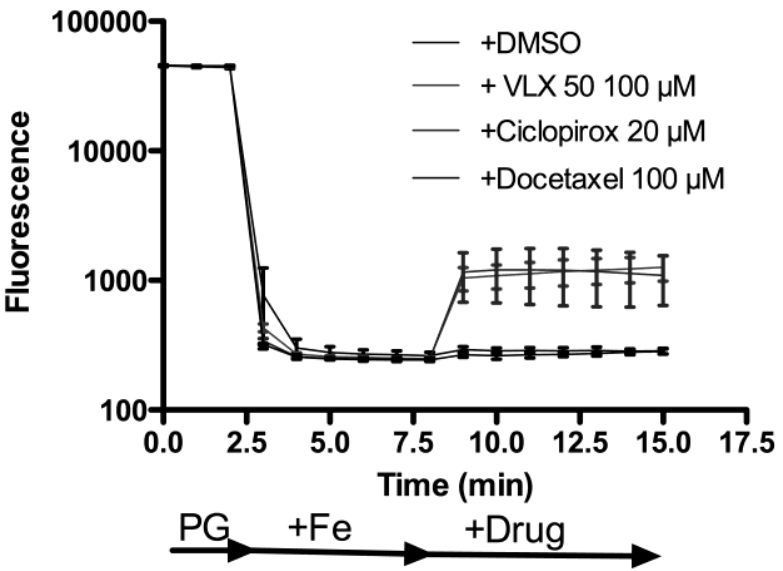


Figure 6

A

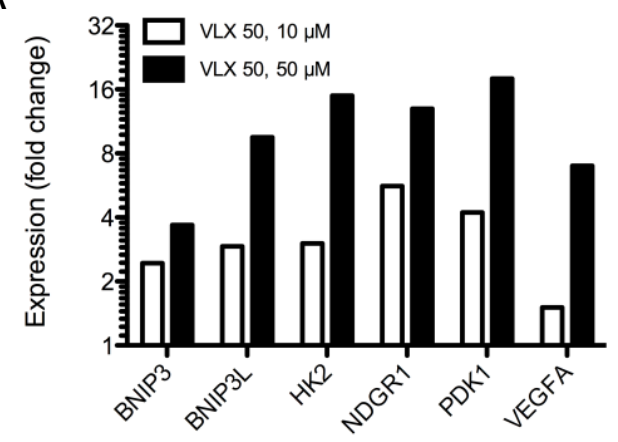

C

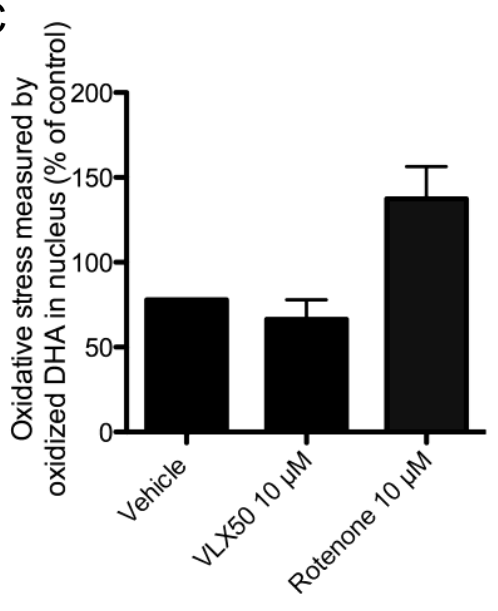

B

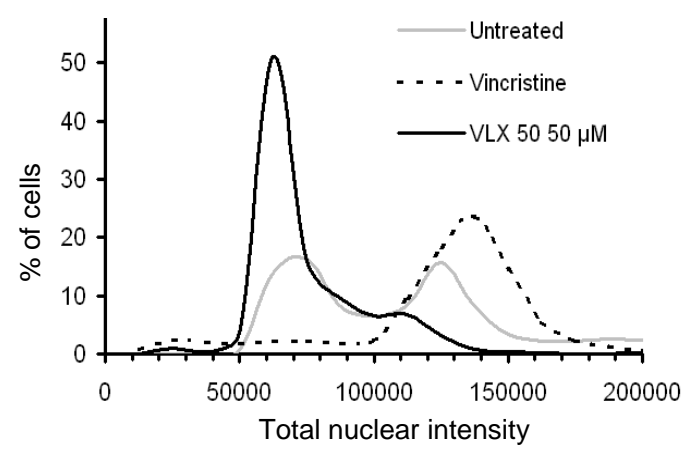

D

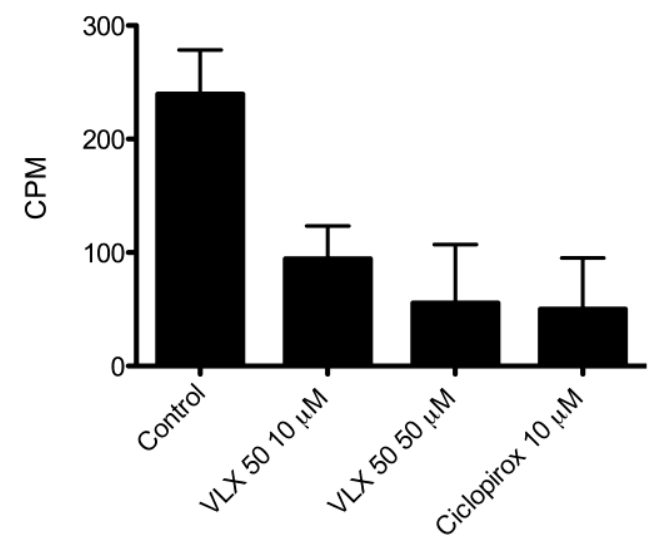

